\title{
Postoperative Logen-Druckmessung der lumbalen Paraspinalmuskulatur
}

\author{
G.Farkas \#, E.Berkman *, W.Dick \# \\ \# Orthopädische Universitätsklinik, Felix Platter-Spital, 4012 Basel \\ * Orthopaedic surgery, 1 Memorial Southwest, 7777 Southwest Freeway, Houston, Texas 77074
}

\section{EINLEITUNG:}

Ziel dieser prospektiven Studie war, die Hypothese zu belegen, dass bekannte degenerative Muskelveränderungen der lumbalen paravertebralen Muskulatur, wie sie nach Wirbelsäulen-Operationen auftreten können, aufgrund hoher postoperativer Compartmentdrücke entstehen $\{10\}$.

$\mathrm{Zu}$ erwarten waren die hohen Druckwerte vor allem in den ersten zwei postoperativen Tagen, wo durch Hämatome und Ödeme eine intrakompartimentelle Volumenzunahme entsteht $\{5,8\}$.

Einerseits spricht man definitionsgemäss von einem Compartment-Syndrom, wenn der Compartmentdruck über $30 \mathrm{mmHg}$ steigt $\{1,3,4,7,9\}$, und andererseits, wenn die Druckdifferenz zwischen diastolischem Blutdruck und Compartmentdruck unterhalb $30 \mathrm{mmHg}$ sinkt $\{7,12\}$. Zudem spielt der Faktor Zeit eine grosse Rolle, denn zunächst noch gut verträgliche Druckwerte können nach einer längeren Zeit (mehr als $8 \mathrm{Std}$.) zu einem Compartment-Syndrom führen $\{3,4,6\}$.

\section{MATERIALMETHODE:}

21 Patienten wurden auf dieselbe Art und Weise gemessen und ausgewertet, pro Patient insgesamt 4 Messungen. Die erste Messung erfolgte unmittelbar vor Hautschnitt, die zweite unmittelbar nach Operations-Ende, die dritte 24 Std. postoperativ und die vierte 48 Std. postoperativ. Aus praktischen Gründen (Lagerung auf dem Op-Tisch) erfolgte die präoperative Messung in Bauchlage, während die anderen drei Messungen in gehaltener Seitenlage durchgeführt wurden.
Zur Messung wurde das gängige Mess-System nach Witschger/Wegmüller verwendet $\{13\}$. Ein steriler Periduralkatheter wurde auf Höhe des vierten Lendenwirbels in der paravertebralen Muskulatur positioniert und mit steriler physiologischer Kochsalzlösung aufgefüllt. Diese Flüssigkeitssäule leitete den Logendruck zu einem Transducer (mit einer Empfindlichkeit von $5 \mathrm{Mikrovolt} / \mathrm{Volt} / \mathrm{mmHg}$ ) von dem dann der elektronische Monitor (mit Selbstkalibrierung) gespiesen wurde.

\section{RESULTATE:}

Die pröoperativen Werte (Messung 1) bewegten sich in der Norm zwischen -5 und $+15 \mathrm{mmHg}\{2,5,13\}$. In der Messung 2 fanden wir bereits vier Patienten mit erhöhten Werten über $30 \mathrm{mmHg}$. Bei zwei dieser Patienten blieb auch Messung 3 über $30 \mathrm{mmHg}$ und bei einem sogar die letzte Messung. Bei Messung 3 zeigten dann acht Patienten (also 38\%) Druckwerte über $30 \mathrm{mmHg}$ und bei Messung 4 waren es sechs (also 29\%). Insgesamt erreichten also 57\% der Patienten einen Druckwert über $30 \mathrm{mmHg}$ und $43 \%$ über $40 \mathrm{mmHg}$.

Die relativen Druckwerte lagen ebenfalls häufig im pathologischen Bereich. Die Differenz des Compartmentdruckes zum diastolischen Blutdruck bewegte sich bei acht Patienten mindestens einmal unter $30 \mathrm{mmHg}$. Vier erreichten mindestens zweimal eine derart niedrige Druckdifferenz.

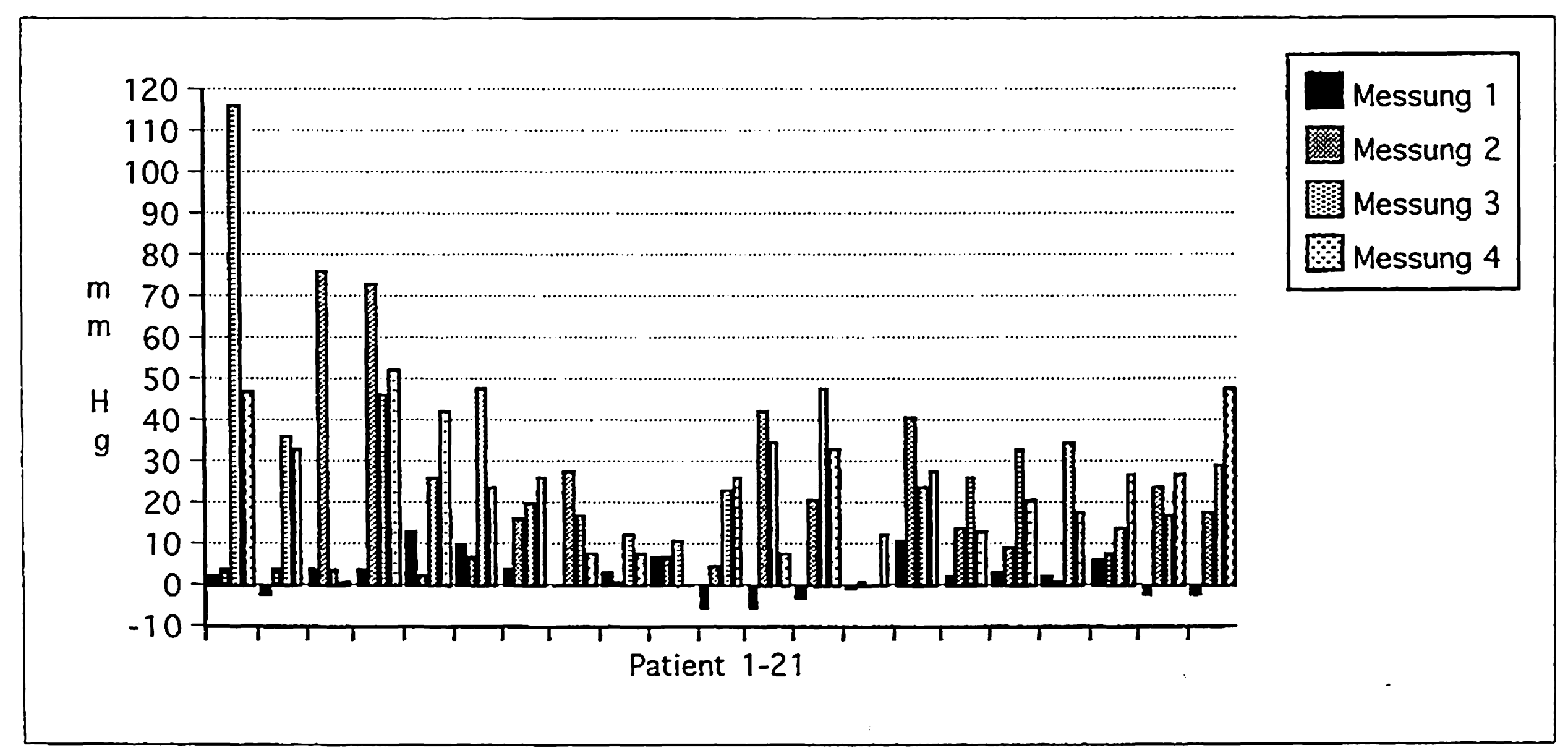



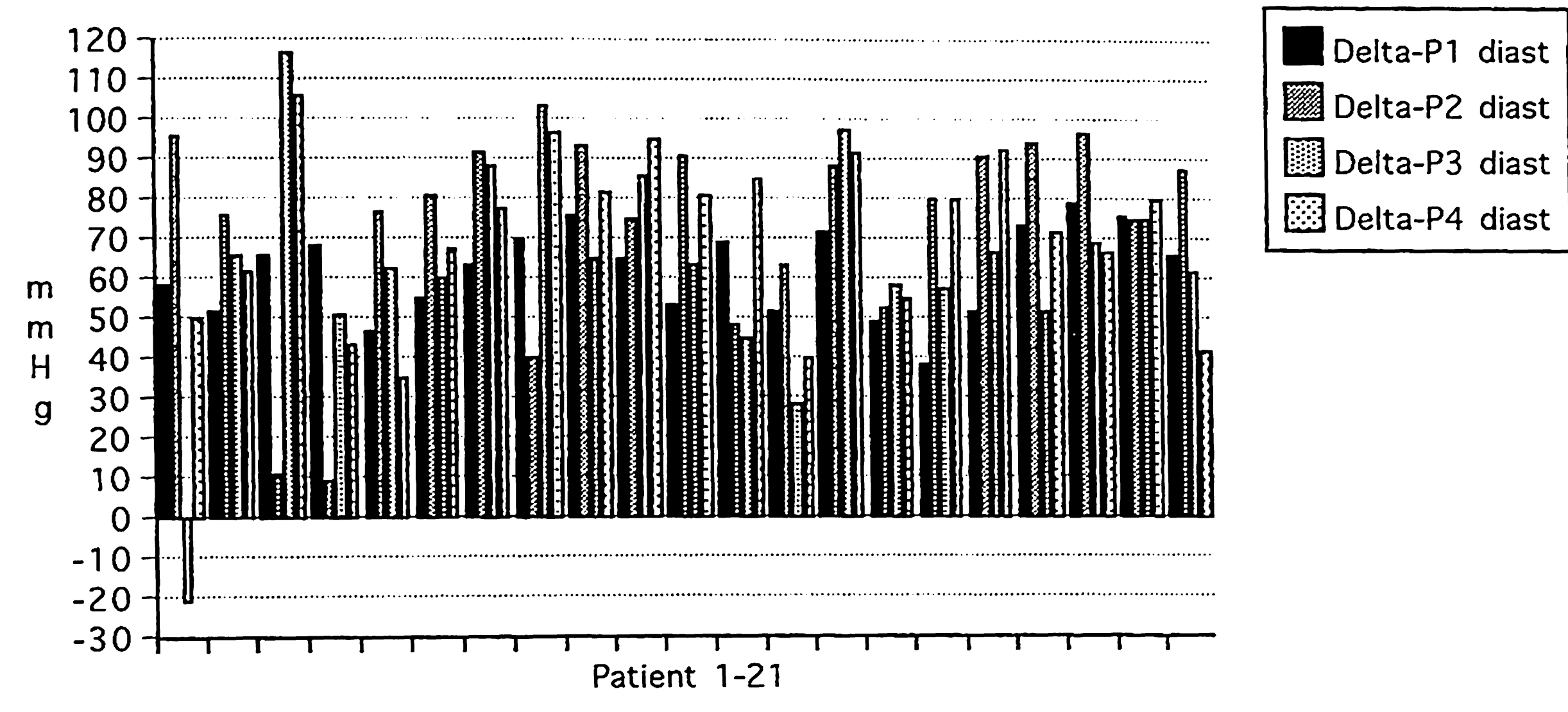

\section{DISKUSSION:}

Wir konnten mit unserer Studie zeigen, dass mehrere unserer Patienten hohe Druckwerte in der ersten postoperativen Phase entwickeln, wie auch geringe Differenzwerte zwischen diastolischem Blutdruck und Compartmentdruck, und dies wahrscheinlich auch uber einen längeren Zeitraum. Somit erfüllen sie die Kriterien eines Compartment-Syndromes. Den genauen Druckverlauf uber die Zeit kennen wir allerdings nicht, was nur mittels einer kontinuierlichen Messung nachweisbar wäre.

\section{LITERATUR:}

1.Bourne RB; Rorabeck CH

"Compartment syndrome of the lower leg"

Clin Orthop, 1989, 240, 97-104

\section{Carr D; Gilbertson L; Krag M; Pope M}

"Lumbar paraspinal compartment syndrome."

SPINE, 1986, 10(9), 816-820

3. Gerow G; Matthews B; Jahn W; Gerow R "Compartment syndrome and shint splint of the leg" J Manipulative Physiol Ther, 1993, 16(4), 245-52

4. Hargens AR; Schmidt AA; Evans KL

"Quantitation of skeletal-muscle necrosis in a model compartment syndrome"

J Bone Joint Surg (A) 63:631-61981

5.Konno S; Kikuchi S; Nagaosa Y

"The relationship between intramuscular pressure of the paraspinal muscles and low back pain"

SPINE, 1994, 19(19), 2186-9
6. Matsen FA; Winquist RA; Krugmire RB

"Diagnosis and management of compartment syndromes"

J Bone Joint Surg (A), 1980, 62(2), 286-91

7. Mc Queen MM; Court-Brown CM

"Compartment monitoring in tibial fractures"

J Bone Joint Surg (B), 1996, 78(1), 99-104

8. Peck D; Nicholls PJ; Beard C; Allen JR

"Are there compartment syndromes in some patients with idiopathic back pain?"

SPINE, 1986, 11(5), 468-75

9.Pedowitz RA; Hargens AR; Mubarak SJ

"Modified criteria for the objektive diagnosis of chronic compartment syndrome of the leg."

Am J Sports Med, 1990, 18(1), 35-40

10. Sihvonen T; Herno A; Paljärvi L; Airaksinen O

"Local denervation atrophy of paraspinal muscles in postoperative failed back syndrome"

SPINE, 1993,18(5), 575-81

\section{Styf J; Lysell E}

"Chronic compartment syndrome in the erector spinae muscle."

SPINE, 1987, 12(7), 680-2

12. Whitesides TE, Haney TC, Morimoto $K$, Harada $H$

"Tissue pressure measurements as a determinant for the need of fasciotomie"

Clin Orthop, 1975, 113, 43-50

13. Witschger PM; Wegmüller $M$

"Apparative Muskeldruckmessung beim akuten und chronischen Compartmentsyndrom"

Z.Unfallchir.Vers.med., 1994, 87(1), 45-51 